12

\title{
Каскадный трансформатор на основе объемного витка для передачи мощности под высокое напряжение
}

\author{
(C) A.П. Денисов, ${ }^{1}$ B.B. Пархомчук,, B.Б. Рева, ${ }^{1}$ A.A. Путьмаков, ${ }^{1}$ J. Li, ${ }^{2}$ L.J. Mao, ${ }^{2}$ M.T. Tang, ${ }^{2}$ \\ H. Zhao, ${ }^{2}$ X.M. Ma, ${ }^{2}$ X.D. Yang ${ }^{2}$ \\ ${ }^{1}$ Институт ядерной фризики им. Г.И. Будкера, \\ 630090 Новосибирск, Россия \\ ${ }^{2}$ Institute of Modern Physics, Chinese Academy of Sciences, \\ 730000 Lanzhou, China \\ e-mail: A.P.Denisov@inp.nsk.su
}

(Поступило в Редакцию 20 сентября 2017 г. В окончательной редакции 25 декабря 2017 г.)

Для ускорительного комплекса HIAF, разрабатываемого в IMP (Китай), предложено использовать систему электронного охлаждения для повышения эффективности инжекции ионов в ускоритель и уменьшения разброса импульсов ионов в пучке. Электронное охлаждение ионного пучка осуществлялось за счет взаимодействия ионов с непрерывным электронным пучком с током до $3 \mathrm{~A}$, энергией до $450 \mathrm{keV}$ и стабильностью энергии на уровне $10^{-4}$ или лучше. Рекуперация энергии электронного пучка осуществлялась за счет источника питания мощностью $5-15 \mathrm{~kW}$, расположенном в вершине высоковольтной колонны - высоковольтном терминале. Рассмотрена работа прототипа системы передачи мощности на основе каскадного трансформатора с объемным витком. Такой трансформатор обладает относительно низкой индуктивностью рассеяния, что позволяет существенно уменьшить количество конденсаторов для ее компенсации. Показано, что такая конструкция позволила без особых проблем передавать до $40 \mathrm{~kW}$ мощности при малых габаритах трансформатора и тепловыделении в трансформаторе не более $10 \mathrm{~kW}$.

DOI: 10.21883/JTF.2018.08.46317.2499

\section{Введение}

Высокоинтенсивный ускорительный комплекс (HIAF - High Intensity heavy ion accelerator facility), разрабатываемый в Институте Современной Физики в Китае (IMP - Institute of Modern Physics) нацелен на производство высокоинтенсивных ионных пучков для широкого диапазона экспериментов в ядерной физике, атомарной физике и для других применений [1]. Комплекс состоит их двух колец. Синхротронное кольцо бустера с магнитной жесткостью $34 \mathrm{~T} \cdot \mathrm{m}$ используется для накопления различных ионов, а спектрометрическое кольцо с жесткостью $13 \mathrm{~T} \cdot \mathrm{m}$ предназначено для проведения экспериментов. В качестве инжектора в бустер используется линейный ускоритель со сверхпроводящим ионным источником на электронно-циклотронном резонансе. Схематическое изображение комплекса НIAF представлено на рис. 1. Сверхпроводящий ионный источник на электронно-циклотронном резонансе (SECR) генерирует высоко заряженные ионные пучки, которые ускоряются в линейном ускорителе (iLinac) с соотношением заряд-масса $Z / A=1 / 7$ (например, ${ }^{238} \mathrm{U}^{34+}$ ) до энергии $17 \mathrm{MeV} / \mathrm{u}$. После прохождения линейного ускорителя ионы будут накапливаться и ускоряться в кольце бустера (BRing) соответственно до требуемых интенсивности и энергии, и затем быстро выводиться из него на неподвижную мишень, расположенную у входа в сепаратор фрагментов (HFRS), для производства редких вторичных изотопов или ионов с более высоким зарядом. Полученные частицы будут использоваться в физических экспериментах в спектрометрическом кольце (SRing). Кроме этого, кольцо бустера Bring планируется оснастить системой медленного вывода пучка для широкого спектра прикладных исследований в биологии и материаловедении.

Для улучшения параметров ионных пучков в обоих кольцах (SRing и BRing) планируется использовать метод электронного охлаждения [2]. В основе метода электронного охлаждения лежит „теплообмен“ между пучком ионов, циркулирующим в накопителе, и пучком электронов, движущимся с той же средней скоростью, что и охлаждаемый ионный пучок. На общем участке траектории в системе покоя пучков „горячие“ протоны охлаждаются за счет кулоновских столкновений в газе „холодных“ электронов. Процесс охлаждения продолжается до тех пор, пока температура протонов в системе центра масс не станет равной эффективной температуре электронного пучка.

B кольце BRing комбинация быстрого электронного охлаждения (энергия электронного пучка $50-120 \mathrm{keV}$ ) и поперечной точечной инжекции пучка позволяет накапливать ионы до интенсивностей, ограниченных эффектами пространственного заряда. Система электронного охлаждения для кольца SRing, рассчитанная на энергии пучка электронов $250-450 \mathrm{keV}$, спроектирована как многофункциональное устройство, которое может быть использовано в различных режимах эксплуатации. В режиме внутренней мишени непрерывный электронный пучок применяется к накапливаемому ионному пучку для компенсации эффектов нагрева от внутренней мише- 


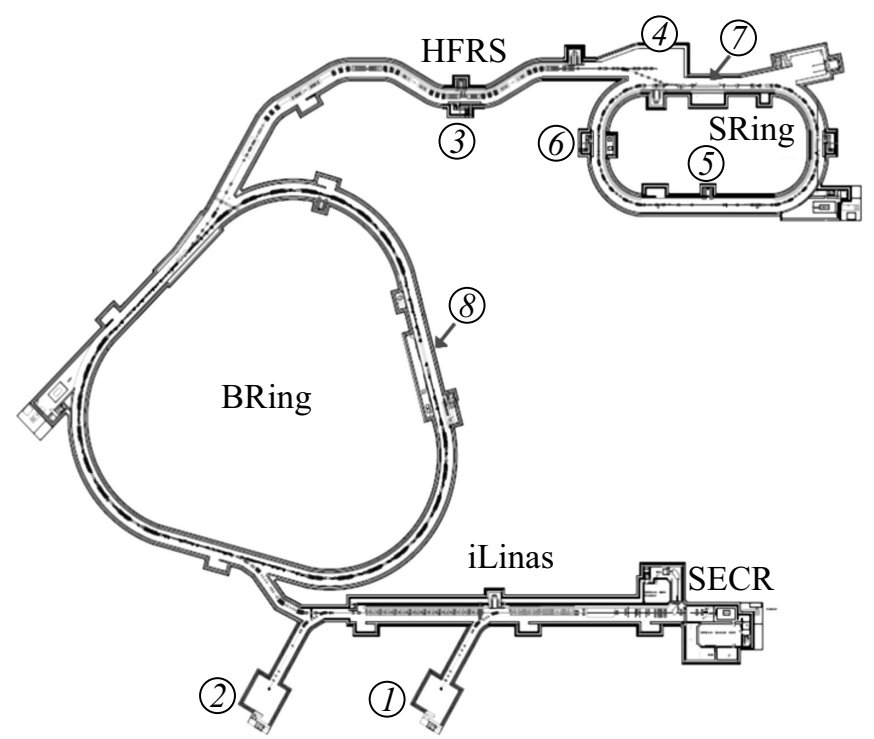

Рис. 1. Общая схема ускорительного комплекса HIAF: 1 спектрометр для изучения структуры ядра на низких энергиях, 2 - экспериментальная станция для облучения пучком ионов, 3 - сепаратор фрагментов, 4 - внешняя экспериментальная станция, 5 - спектрометрическое кольцо, 6 - диэлектронный рекомбинационный спектрометр, 7, 8 - системы электронного охлаждения для SRing и BRing соответственно.

ни. В нормальном режиме работы используется комбинация электронного и стохастического охлаждения, что позволяет значительно уменьшить время охлаждения, особенно пучков радиоактивных ионов, образующихся при прохождении фиксированной мишени и обладающих большим разбросом поперечных и продольных импульсов.

Общая схема системы электронного охлаждения для SRing приведена на рис. 2. Установка электронного охлаждения включает в себя электронную пушку, коллектор, магнитную и вакуумную системы, систему питания. Она спроектирована по образцу существующих электронных охладителей в IMP, произведенных в Институте ядерной физики им. Будкера в 2002 г. [3-6].

Основой для ускорения электронов до требуемой энергии в установке электронного охлаждения SRing является высоковольтная колонна (рис. 2, 3). Она включает в себя устройство генерации высокого напряжения и источник питания коллектора, расположенный на вершине высоковольтной колонны (высоковольтном терминале), необходимый для обеспечения рекуперации энергии электронного пучка. Высоковольтная колонна включает в себя систему генерации высокого напряжения, систему распределения мощности между устройствами высоковольтной колонны, пушку и коллектор. Электронный пучок формируется в электронной пушке (рис. 3,1 ), затем ускоряется в электростатическом ускорителе прямого действия до требуемой энергии (рис. 3,3) и на тороидальном участке (рис. 2,4) совмещается с ионной орбитой. На участке охлаждения (рис. 3,5) происходит взаимодействие электронного и ионного пучков. Далее электронный пучок покидает ионную орбиту, замедляется (рис. 3,4 ) и поглощается в коллекторе (рис. 3, 2).

Основное потребление мощности приходится на рекуперацию энергии электронного пучка и определяется током электронного пучка, а также напряжением между катодом электронной пушки и коллектором электронов (напряжение рекуперации). Данное напряжение задается при помощи источника питания коллектора (типичное значение $5 \mathrm{kV}$ ), размещенного в высоковольтном терминале. При использовании непрерывного электронного пучка с током 2 А источник питания коллектора потребляет до $10 \mathrm{~kW}$ мощности. Также стоит отметить, что без использования рекуперации энергии электронного пучка потребляемая мощность определялась бы полным напря-

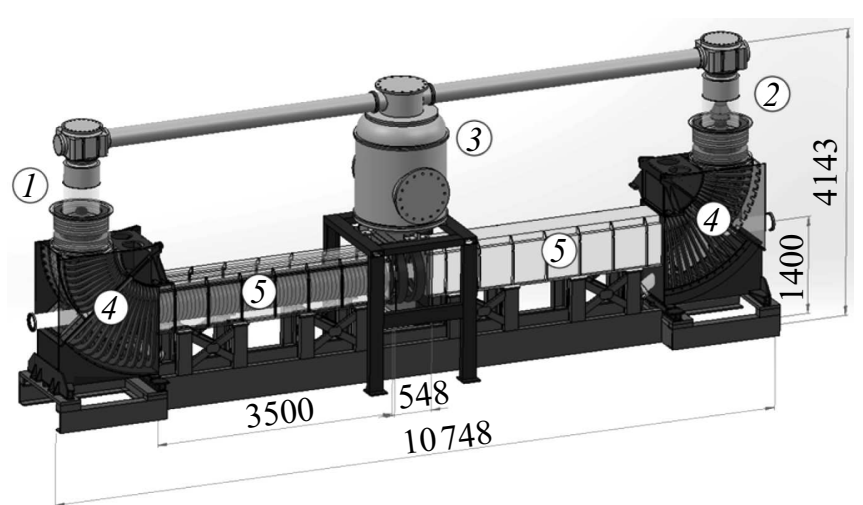

Рис. 2. Общая схема установки электронного охлаждения для SRing: 1 - электронная пушка, 2 - коллектор электронов, 3 - высоковольтный бак, 4 - поворотные магниты, 5 соленоид секции охлаждения.

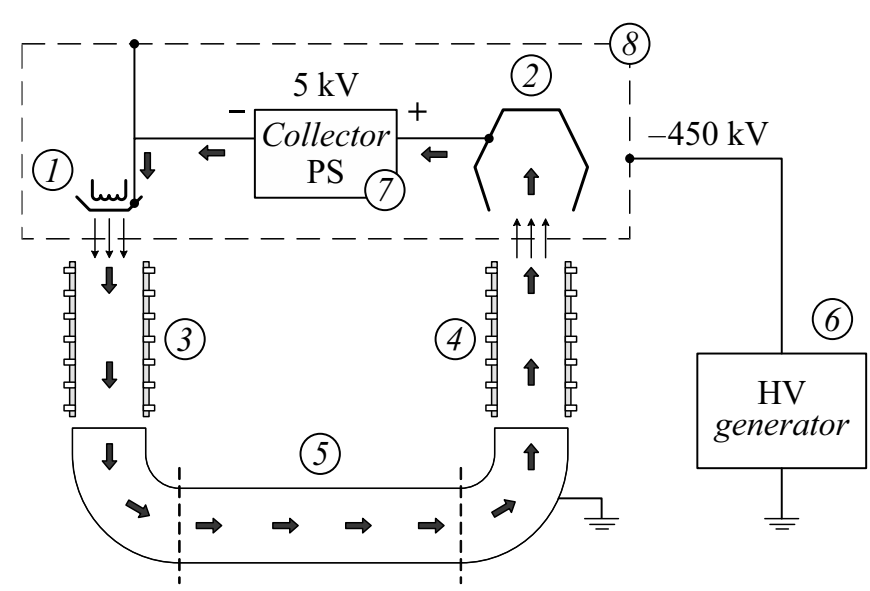

Рис. 3. Принципиальная схема устройства высоковольтной системы электронного охладителя: 1 - катод электронной пушки, 2 - коллектор электронов, 3, 4 - ускорительные трубки для ускорения и торможения электронов соответственно, 5 - секция охлаждения, $6-$ источник высокого напряжения для ускорения/торможения электронного пучка, 7 - источник питания коллектора, 8 - высоковольтный терминал. 
жением высоковольтной колонны $450 \mathrm{kV}$ и составляла бы $900 \mathrm{~kW}$.

Для передачи мощности в высоковольтной колонне системы электронного охлаждения планируется использовать каскадный трансформатор, который способен передавать мощность от секции к секции при помощи переменного магнитного поля, обеспечивая тем самым гальваническую развязку устройств высоковольтного терминала от внешнего источника питания. Большая связь по потоку между обмотками каскадного трансформатора позволяет с высокой эффективностью передавать мощность от секции к секции. А за счет того, что для высоковольтной системы электронного охладителя для SRing не требуется большого числа секций трансформатора, можно перейти к конструкции трансформатора на основе объемного витка.

В настоящей работе была поставлена задача изучить работу каскадного трансформатора на основе объемного витка, а также определить максимальную мощность, которую такой трансформатор способен передавать.

\section{1. Способы передачи мощности в высоковольтной системе}

Существует несколько способов решения проблемы передачи электрической мощности к устройствам, расположенным под высоким потенциалом. Рассмотрим некоторые из них, применимые к установкам электронного охлаждения.

В установках с энергией до $300 \mathrm{keV}$, как правило, применяют системы передачи мощности, выполненные по трансформаторному принципу. А именно это либо обычные электротехнические трансформаторы с усиленной изоляцией между обмотками, расположенные на едином магнитопроводе, либо трансформаторы с большим зазором между обмотками, когда часть пути магнитный поток проходит вне магнитопровода по изолирующей среде [7]. Такая схема передачи мощности используется на установке электронного охлаждения ЭХ-300, созданной в ИЯФ СО РАН для ускорительного комплекса IMP $[8,9]$. В нем первичная и вторичная обмотки намотаны на коаксиальные поверхности, образующие высоковольтную линию для передачи напряжения к пушке и коллектору электронов. В такой конструкции большой зазор приводит к большому значению индуктивности рассеяния, что негативно сказывается на нагрузочной характеристике мощного выпрямителя. Резкое изменение нагрузки, характерное для установок электронного охлаждения вследствие срыва рекуперации пучка, приводит к большим изменениям напряжения на вторичной обмотке. Кроме этого, большая величина переменных магнитных полей вне магнитопровода способна наводить электрические поля на элементах конструкции высоковольтного терминала, затрудняя достижения требуемой стабильности энергии электронного пучка.
Развивая идею об использовании трансформатора без сердечника, можно разбить вторичную обмотку на независимые обмотки, расположенные внутри одной первичной обмотки. Каждая вторичная обмотка входит в состав секции, включающей конденсатор, или несколько конденсаторов, для компенсации индуктивности рассеяния, и выпрямитель. Далее часть мощности с каждой секции может быть использована для собственных нужд устройств, входящих в состав этой секции, а другая часть мощности может быть передана в область высоковольтного терминала. По этому принципу работают ускорители электронов типа ЭЛВ [10] с характерной рабочей частотой 100-1000 Hz. Преимуществами данной схемы являются улучшенная электрическая изоляция между секциями и значение максимальной мощности, которую можно передавать (около $500 \mathrm{~kW}$ ). Каждая секция также может быть использована в качестве источника высокого напряжения, однако без использования дополнительных выпрямителей и сложных систем обратной связи стабильности этого напряжения недостаточно для целей электронного охлаждения.

Несмотря на это, подобное разделение колонны на отдельные секции дает возможность конструктивно объединить систему генерации высокого напряжения и систему силового питания. Индуктивная связь дает возможность передавать большую мощность, часть из которой можно отбирать с отдельных секций для нужд высоковольтных источников. Однако описанная выше схема, в которой отдельные секции связываются со внешней катушкой индуктивности, имеет малую связь по потоку. Возможным решением является использование каскадного трансформатора, в котором каждая секция связана по магнитному потоку только с примыкающими к ней секциями. Такая схема передачи мощности используется в установке электронного охлаждения для COSY [11]. Каскадный трансформатор для электронного охладителя в COSY состоит из 33 секций, разделенных потенциалом $60 \mathrm{kV}$, и рассчитан на передачу мощности $20 \mathrm{~kW}$ к высоковольтному терминалу и по $500 \mathrm{~W}$ на каждую отдельную секцию для питания магнитной системы и системы генерации высокого напряжения. Каждый каскад включает в себя трансформатор с первичной и вторичной обмоткой, а также обмоткой отбора мощности, намотанных на сердечник с большим показателем магнитной проницаемости $(\mu \approx 30000)$. Рабочая частота трансформатора составляет $25 \mathrm{kHz}$, что допускает использование кольцевого магнитопровода с внешним диаметром $0.28 \mathrm{~m}$ и сечением $2 \times 4 \mathrm{~cm}$, что существенно меньше, чем требуется для работы на низких частотах. Полная высота каскадного трансформатора $2 \mathrm{~m}$, а диаметр внешнего корпуса $0.38 \mathrm{~m}$. Большая длина трансформаторной колонны, а следовательно, и большие изоляционные промежутки $30 \mathrm{~mm}$ между секциями трансформатора позволили достигнуть потенциал высоковольтного терминала $1.6 \mathrm{MV}$. Использование большого числа секций с независимыми умножителями напряжения для создания высокого напряжения поз- 
волило достичь пульсаций энергии электронов лучше, чем $50 \mathrm{~V}$ на уровне $1 \mathrm{MV}$, что было установлено по результатам измерений на протонном пучке.

При большом количестве секций трансформатора и высокой частоте входного сигнала просадка напряжения из-за наличия рассеянного потока в секциях становится существенной. Каждая секция трансформатора оснащается конденсаторами для компенсации индуктивности рассеяния, что делает сложной как саму конструкцию трансформатора (расположение многочисленных элементов в секции и обеспечения изоляции между ними), так и процесс его сборки. В случае если трансформатор имеет малое число секций, то просадка напряжения из-за рассеянного магнитного потока становится менее существенной. Более того, индуктивность рассеяния секций может быть уменьшена за счет выбора более оптимальной схемы расположения витков обмоток вдоль секции. В случае высоковольтной колонны, рассчитанной на $450 \mathrm{kV}$ и состоящей всего из 10 секций, вопрос о возможности упрощения схемы и конструкции трансформатора представляет особый интерес, а именно о том, какую максимальную мощность возможно передать через малосекционный трансформатор без использования компенсирующих элементов в каждой его секции.

Кроме вышеуказанных электротехнических методов передачи мощности стоит упомянуть и механические, которые были использованы в действующих установках. Например, в установке электронного охлаждения в Fermilab для передачи мощности использовался вращающийся диэлектрический вал. В нижней части высоковольтной колонны располагался мотор, вращающий вал, а в вершине колонны находился генератор, преобразующий энергию вращения в электрическую [12]. Значение максимальной передаваемой мощности данного устройства составляло $18 \mathrm{~kW}$, при напряжении высоковольтного терминала $4.3 \mathrm{MV}$. Основными сложностями, с которыми пришлось столкнутся авторам этого метода, это необходимость высокоточного закрепления диэлектрического вала и наличие механических колебаний от его вращения [12]. Вышеуказанные проблемы могут быть решены, если вместо вращения вала использовать механическую энергию потока сжатого газа, которая преобразуется в электрическую посредством пропускания потока газа через турбогенератор [13]. Данный способ успешно используется на ускорительном масс-спектрометре, произведенном в ИЯФ СО РАН, с энергией пучка $1 \mathrm{MeV}$ [14].

\section{2. Каскадный трансформатор без компенсации индуктивности рассеяния в каждой секции}

Схема каскадного трансформатора заметно упрощается, если секции трансформатора не содержат элементов компенсации индуктивности рассеяния, а именно соседние секции становятся связанными только через

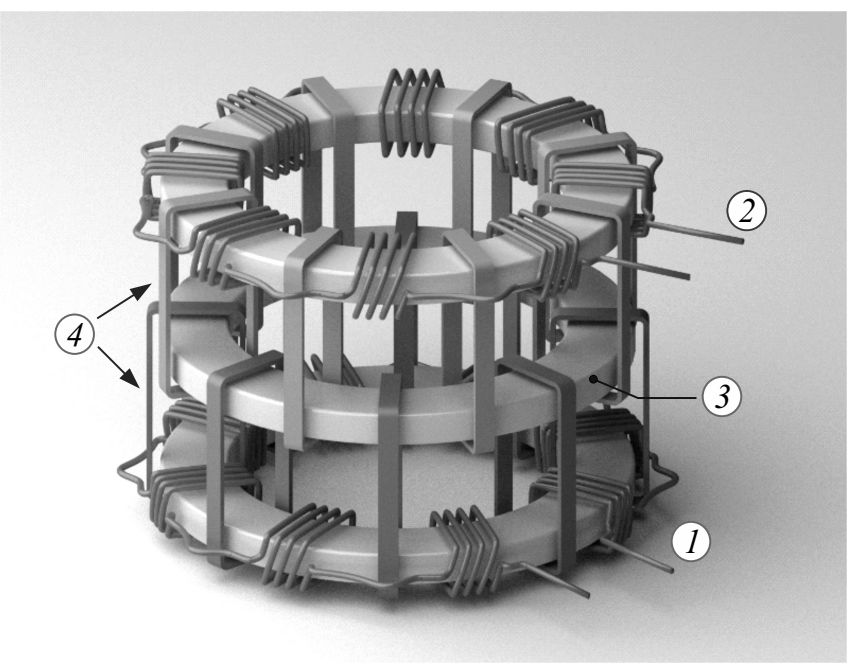

Рис. 4. Конструкция каскадного трансформатора: 1,2 - входная/выходная обмотки трансформатора, 3 - магнитопровод, 4 - короткозамкнутые витки связи между секциями трансформатора.

обмотки. Индуктивность рассеяния обмоток трансформатора может быть уменьшена путем равномерного распределения витков обмоток вдоль магнитопровода. Это следует из простых соображений о том, что если витки вторичной обмотки совпадают по расположению с соответствующими витками первичной обмотки, то индуктивность рассеяния равняется нулю, а при максимальном удалении обмоток друг от друга индуктивность рассеяния максимальна. Так как первичная и вторичная обмотки каждой секции трансформатора разнесены по потенциалу, что обусловлено требованием к распределению потенциала вдоль трансформаторной колонны, то минимальное расстояние между витками первичной и вторичной обмоток ограничено изоляционным расстоянием. В этом случае равномерное распределение витков первичной и вторичной обмоток вдоль магнитопровода, чередующихся между собой, соответствует наиболее близкому расположению витков первичной и вторичной обмоток при обеспечении необходимых изоляционных промежутков.

Поэтому было предложено заменить группы витков отдельными независимыми короткозамкнутыми витками, обеспечивающими связь между соседними секциями. В данном случае в качестве первичной и вторичной обмоток секции выступают одинарные витки, а индуктивность рассеяния секции трансформатора уменьшена за счет расположения этих витков равномерно вдоль магнитопровода. Расположение витков связи и магнитных элементов каскадного трансформатора представлено на рис. 4 на примере каскада из трех секций.

Входная обмотка нижней секции и выходная обмотка верхней секции состоят из большого числа витков, что необходимо для ограничения тока холостого хода трансформатора. Соседние секции связаны друг с другом 
посредством восьми витков, распределенных равномерно вдоль кольца магнитопровода. Виток связи в данной конструкции является одновременно вторичной обмоткой для одной секции и первичной обмоткой для другой. По принципу связи между кольцами магнитопровода данный трансформатор близок к трансформатору с объемным витком. Однако есть и существенное отличие наличие промежутков в расположении короткозамкнутых витков, необходимых для каскадного соединения соседних секций друг с другом. Конструктивно виток связи представляет из себя медную рамку, охватывающую сердечники двух соседних секций. Изоляция медных витков от магнитопровода осуществляется при помощи специальных вставок из капролона.

В установке электронного охлаждения для SRing каскадный трансформатор будет иметь 10 секций (рис. 5). Параметры данного трансформатора приведены в табл. 1. Кольца трансформатора располагаются в специальном трансформаторном баке, являющемся частью высоковольтной колонны, также разделенной на секции. Каждой секции трансформатора соответствует отдельная секция колонны, при этом соседние секции колонны разведены по потенциалу и изолированы друг от друга керамическими кольцевыми изоляторами. Керамические изоляторы вместе с основаниями секций образуют закрытый объем, в котором расположен трансформатор и через который пропускается охлаждающая жидкость (масло или кремнийорганическая жидкость) для повышения электрической прочности и охлаждения обмоток и магнитопроводов. При испытаниях прототипа на воздухе изоляция между обмотками выдерживала напряжение $10 \mathrm{kV}$, что вполне достаточно при условии погружения обмоток в трансформаторное масло. Вне этого объема в секциях колонны размещается управляющая и силовая электроника, включая источники высокого напряжения.

Основными преимуществами описанной конструкции являются простота сборки и разборки трансформатора, а также возможность распределить витки связи вдоль магнитных сердечников секций, тем самым уменьшив индуктивность рассеяния секций трансформатора и при этом обеспечив необходимую электрическую изоляцию

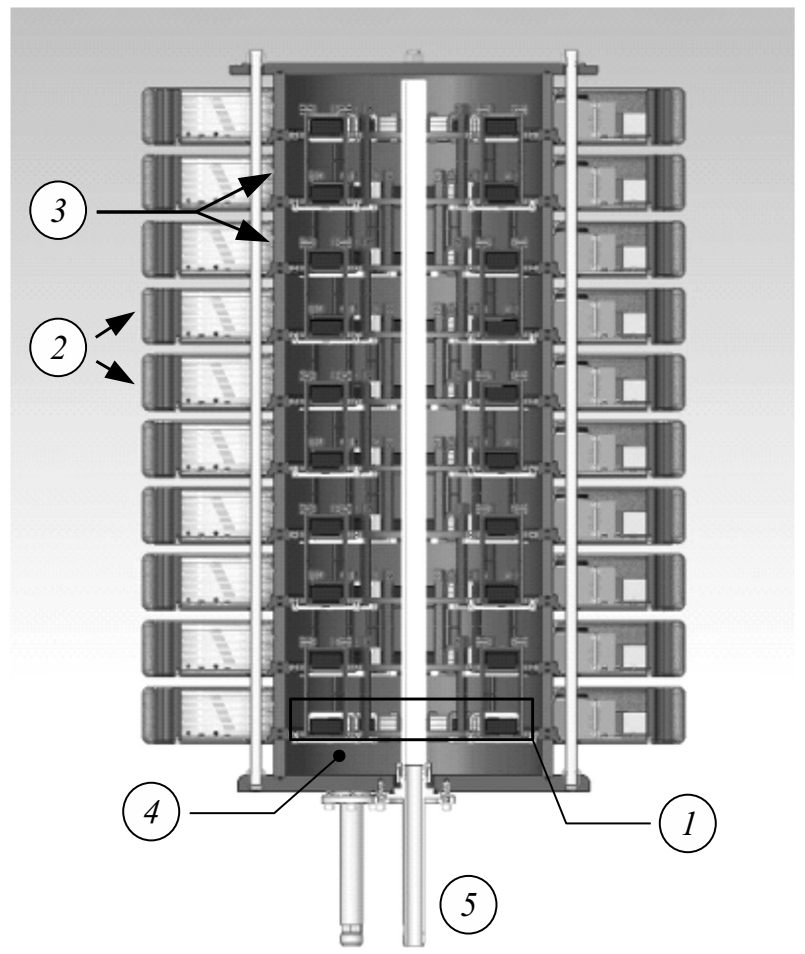

Рис. 5. Конструкция высоковольтной колонны: 1 - секция трансформатора, 2 - секции колонны, разнесенные по потенциалу, с расположенной на них электроникой, 3 - керамические кольца для изоляции соседних высоковольтных секций, 4 - объем, наполняемый охлаждающей жидкостью, 5 - каналы для впуска и выпуска охлаждающей жидкости.

между принимающими и передающими обмотками. Главным неудобством данной конструкции является невозможность компенсации индуктивности рассеяния обмоток связи на каждой секции трансформатора.

\section{1. Параметры секции трансформатора}

Определяющими параметрами каскадного трансформатора при его проектировании для передачи мощности являются индуктивность рассеяния обмоток каждой

Таблица 1. Параметры каскадного трансформатора для системы охлаждения для SRing

\begin{tabular}{l|c}
\hline \multicolumn{1}{c|}{ Параметр } & Описание \\
\hline Кольца магнитопровода & Аморфное железо $(5$ БДСР $)$ \\
Рабочая магнитная индукция & $2 \mathrm{kG}$ \\
Магнитные потери при индукции $0.2 \mathrm{~T}(25 \mathrm{kHz})$ & $12 \mathrm{~W} / \mathrm{kg}$ \\
Внешний диаметр кольца магнитопровода & $0.14 \mathrm{~m}$ \\
Внутренний диаметр кольца магнитопровода & $0.1 \mathrm{~m}$ \\
Толщина магнитопровода & $0.02 \mathrm{~m}$ \\
Расстояние между секциями & $0.09 \mathrm{~m}$ \\
Витки связи между соседними секциями & 8 штук \\
Входная и выходная обмотки трансформатора & 32 витка \\
Высота высоковольтной колонны & $0.9 \mathrm{~m}$ \\
Диаметр высоковольтной колонны & $0.74 \mathrm{~m}$
\end{tabular}




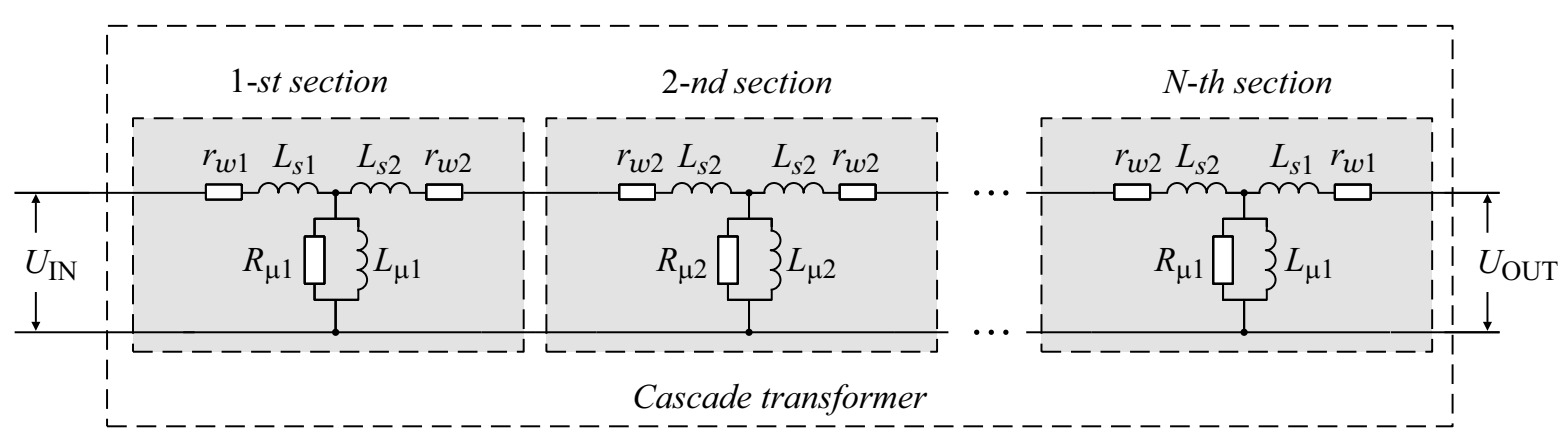

Рис. 6. Эквивалентная схема каскадного трансформатора с секциями, представленными Т-образной схемой. Начальная и конечная секции несимметричны, так как имеют различные первичную и вторичную обмотки.

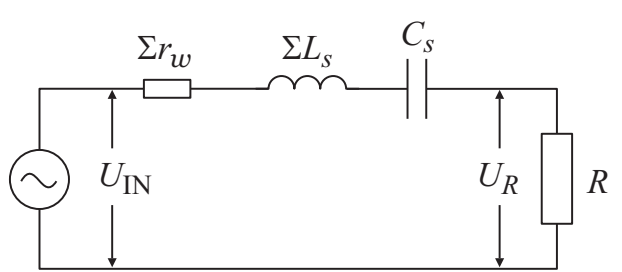

Рис. 7. Схема измерения вклада сопротивления обмоток и индуктивности рассеяния в проходной импеданс трансформатора. $\Sigma r_{w}$ - суммарный вклад сопротивления обмоток, $\Sigma L_{S}-$ сумарный вклад индуктивности рассеяния секций, $C_{S}=1.3 \mu \mathrm{F}$, $R=3.2 \Omega$.

секции $\left(L_{s}\right)$ и сопротивление обмоток секции $\left(r_{w}\right)$, а также индуктивность связи обмоток $\left(L_{\mu}\right)$ и потери в сердечнике на перемагничивание $\left(R_{\mu}\right)$. Эквивалентная схема трансформатора изображена на рис. 6. Каждая секция представлена эквивалентной Т-образной схемой, параметры которой приведены к первичной обмотке начальной секции, т.е. если бы вместо набора витков связи использовались стандартные обмотки, состоящие из того же числа витков, что и входная обмотка трансформатора. Эти параметры могут быть найдены по измерениям АЧХ трансформатора. В случае если требуется знать реальный ток, протекающий через витки связи или напряжение на них, то достаточно воспользоваться коэффициентом трансформации напряжения. Так, например, если входная обмотка трансформатора имеет 32 витка, то значение суммарного тока, протекающего через витки связи, соединяющие две секции, в 32 раза больше значения приведенного тока между этими секциями.

Метод определения параметров секций трансформатора можно качественно изобразить на примере измерений выходного сопротивления трансформатора. Для определения параметров трансформатора были собраны прототипы из двух и трех секций. Первичная обмотка первой секции и вторичная обмотка последней секции имели по 32 витка. Измерения проводились на звуковом генераторе для двух- и трехсекционного прототипа по схеме измерения, представленной на рис. 7. В изме-

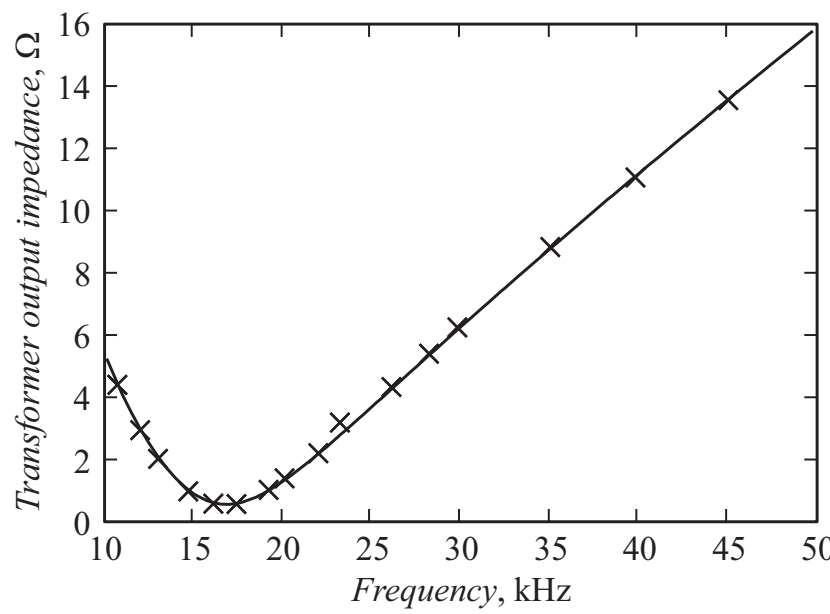

Рис. 8. Зависимость модуля выходного импеданса трансформатора с последовательно включенным конденсатором от рабочей частоты. Перекрестия - измеренные значения для трехсекционного прототипа, линия - моделированные значения.

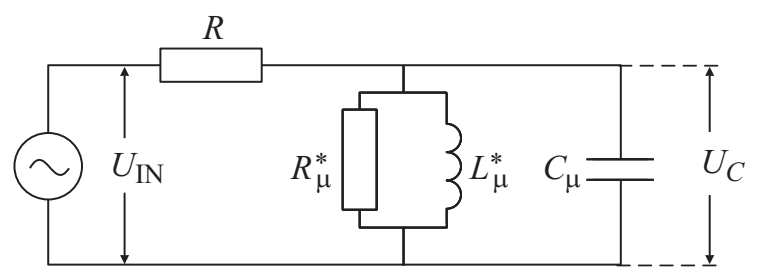

Рис. 9. Схема измерения индуктивности намагничивания секций трансформатора и эквивалентного сопротивления потерь на перемагничивание колец магнитопровода: $R_{\mu}^{*}-$ результирующее сопротивление потерь при параллельном соединении сопротивлений потерь каждой секции, $L_{\mu}^{*}-$ результирующая индуктивность при параллельном включении индуктивностей намагничивания каждой секции, $C_{\mu}=3.5 \mathrm{nF}, R=30 \mathrm{k} \Omega$.

рениях величина емкости подбирается таким образом, чтобы организовать последовательный резонанс на частоте, близкой к $20 \mathrm{kHz}$. Измерительное сопротивление $R=3.2 \Omega$. В данных измерениях влиянием индуктивности связи и потерями пренебрегаем. На примере трех- 
Таблица 2. Измеренные параметры секций трансформатора

\begin{tabular}{l|c|c}
\hline \multicolumn{1}{c|}{\begin{tabular}{c} 
Измеряемый \\
\multicolumn{1}{c}{ параметр }
\end{tabular}} & \multicolumn{2}{|c}{ Измеренные значения, отнесенные к } \\
\cline { 2 - 3 } & крайним секциям & средней секции \\
\hline Сопротивление обмоток & $r_{w 1}=100 \mathrm{M} \Omega$ & $r_{w 2}=79 \mathrm{M} \Omega$ \\
Индуктивность & $L_{s 1}=11.2 \mu \mathrm{H}$ & $L_{s 2}=11.2 \mu \mathrm{H}$ \\
рассеяния & $L_{\mu 1}=45 \mathrm{mH}$ & $L_{\mu 2}=32 \mathrm{mH}$ \\
Индуктивность связи \\
Сопротивление потерь \\
в сердечнике
\end{tabular}

секционного прототипа по измеренной характеристике трансформатора (рис. 8) можно определить вклад сопротивления обмоток в импеданс трансформатора, что соответствует импедансу трансформатора на резонансной частоте. Далее подбирается такое значение индуктивности рассеяния, чтобы модельные значения ложились на измеренную кривую. Исходя из эквивалентной схемы трансформатора, разница между значениями выходного импеданса измеренных двух- и трехсекционного прототипов, есть выходной импеданс центральной секции. На основе полученных значений вычисляются параметры входной и выходной секций.

Аналогичные измерения проводятся для измерения индуктивностей связи секций и сопротивлений потерь в сердечниках. Эквивалентная схема измерений приведена на рис. 9. В данном случае можно пренебречь вкладом индуктивности рассеяния и сопротивлением обмоток. Полученные по результатам данных измерений параметры трансформатора представлены в табл. 2. Результирующее значение индуктивности рассеяния составляет $L_{S}=11 \mu \mathrm{H}$, что в 2 раза меньше, чем аналогичное значение для трансформатора, используемого в электронном охладителе для COSY [11].

\section{2. Силовые испытания}

Для проверки параметров трансформатора, измеренных с использованием генератора звуковой частоты с малой амплитудой напряжения, были произведены эксперименты на прототипе из трех секций с использованием силового источника с рабочей частотой $f=25 \mathrm{kHz}$ и напряжением до $300 \mathrm{~V}$. Схема измерений представлена на рис. 10. Напряжение с секций трансформатора измеряется при помощи одиночных витков. Величина нагрузки могла изменяться в широких пределах, обеспечивая отбор мощности с трансформатора от 0 до $5 \mathrm{~kW}$.

Пример измеренных напряжений на секциях при нагруженном трансформаторе на сопротивление $R_{\mathrm{LOAD}}=$ $=10 \Omega$ представлен на рис. 11. Наличие индуктивности рассеяния приводит к просадке напряжения от секции к секции, а также к сдвигу по фазе сигналов с различных секций. При этом высокочастотные составляющие сигнала проседают сильнее, что видно по сглаживанию заостренных пиков при переходе от секции к секции.
Это обусловлено тем, что вклад от индуктивности в выходной импеданс линейно растет с частотой сигнала. Оценка величины просадки напряжения и сдвига сигналов по фазе может быть произведена из следующего соотношения измеренных напряжений с первой и последней секций:

$$
\frac{U_{3}}{U_{1}}=\frac{1 i \omega L_{S}+R_{\mathrm{LOAD}}}{5 i \omega L_{S}+R_{\mathrm{LOAD}}} .
$$

В (1) учитывается, что $U_{1}$ и $U_{3}$ измеряются не напрямую с передающих обмоток, а с концов измерительных витков. Для оценки полагается, что измеренное напряжение с секции соответствует средним точкам Т-образной эквивалентной схемы. Коэффициенты перед $i \omega$ в числителе и знаменателе показывают сколько раз индуктивность рассеяния $L_{S}$ встречается на участке между нагрузочным сопротивлением $R_{\mathrm{LOAD}}$ и точками измерения напряжения на третьей и первой секциях соответственно. Стоит отметить, что индуктивность рассеяния между измерительным витком и обмоткой, напряжение на которой необходимо измерить, может быть отлична от $L_{S}$, что может вносить небольшую поправку в коэффициенты перед $i \omega$ в (1).

Используя значение $L_{S}$ из табл. 2 и подставляя его в (1), получаем оценку на задержку между сигналами с первой и третьей секций $4 \mu$ s и отношение их амплитуд $\left|U_{3} / U_{1}\right|=0.7$, что хорошо согласуется с измеренными данными $\left|U_{3} / U_{1}\right|_{\mathrm{m}}=0.6$ (рис. 11). Отличие отно-

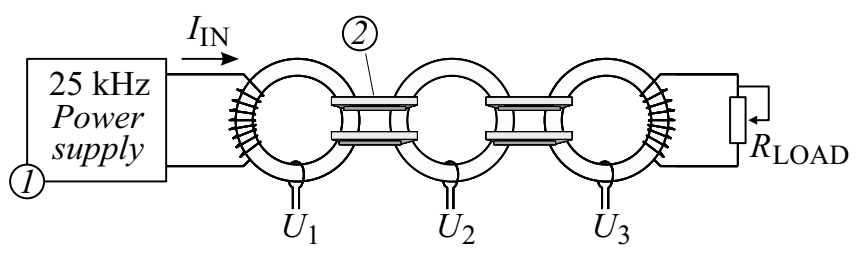

Рис. 10. Схема измерений трехсекционного трансформатора с использованием силового источника питания: 1 - источник синусоидального напряжения, 2 - витки связи между сердечниками соседних секций (восемь параллельных витков).

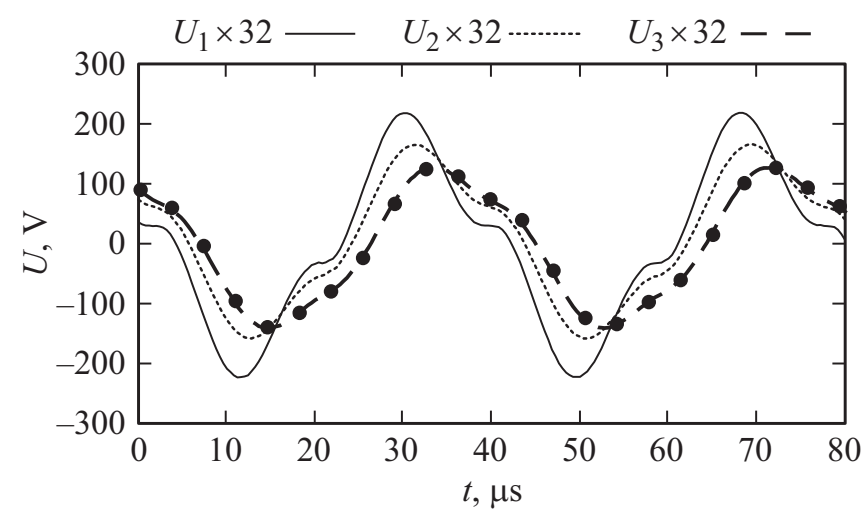

Рис. 11. Измеренные сигналы с секций трансформатора при подключенной напрямую к трансформатору нагрузке $10 \Omega$. Точками отмечены моделированные значения сигнала на третьей секции, опирающиеся на измерения первой секции. 


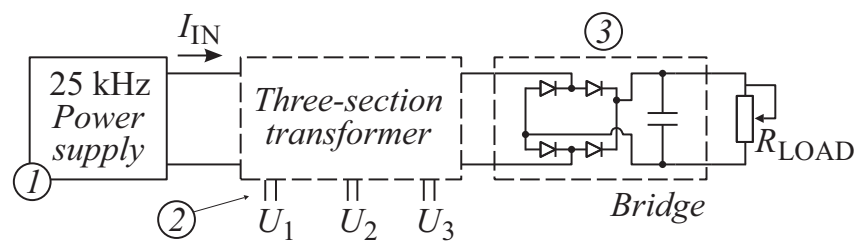

Рис. 12. Схема силовых измерений прототипа трансформатора с выпрямителем, подключенным к его выходу: 1 - силовой источник синусоидального напряжения, 2 - измерительные обмотки с секций трансформатора, 3 - диодный двухполупериодный выпрямитель, конденсатор мостового выпрямителя, Bridge $=1.3 \mu \mathrm{F}$.
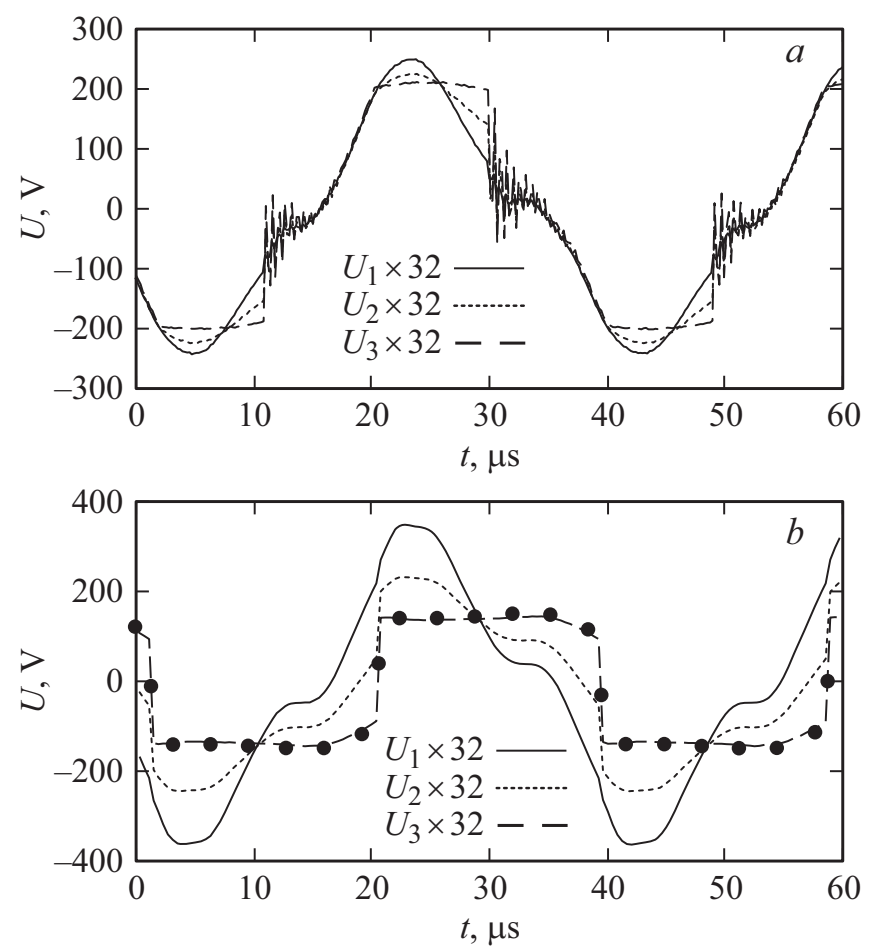

Рис. 13. Формы сигналов, измеренных на различных секциях трансформатора при различной нагрузке, подключенной через двухполупериодный выпрямитель: $a-$ подключенная нагрузка $200 \Omega, b-$ нагрузка $8 \Omega$.

шения амплитуд обусловлено наличием дополнительных гармоник в сигнале. Более высокие частоты подавляются сильнее, поэтому оценка, сделанная при помощи (1), является завышенной.

Измеренный сигнал $U_{1}$ можно разбить на отдельные гармоники, используя фурье-преобразование, и для каждой гармоники при помощи (1) вычислить, как изменяются амплитуда и фаза сигнала при переходе к третьей секции. Затем, применяя обратное фурьепреобразование, можно построить ожидаемую форму сигнала. На рис. 11 жирными точками отмечены моделированные значения напряжения на третьей секции, которые с высокой точностью повторяют форму измеренного сигнала.
Стандартная схема передачи мощности подразумевает наличие двухполупериодного диодного выпрямителя (рис. 12) на выходе каскадного трансформатора в высоковольтном терминале. Как правило, он необходим, так как потребители мощности выпрямляют переменное напряжение, а затем полученное постоянное напряжение преобразуют в импульсы высокой частоты и установленной скважности для осуществления регулировки выходного тока и напряжения.

На рис. 13 представлены формы сигналов, измеренных с секций трансформатора при различной нагрузке, подключенной к выпрямителю. Наличие выпрямителя на выходе трансформатора приводит к тому, что форма сигнала на третьей секции отличается от синусоидальной. Однако из-за индуктивности рассеяния секций сигналы с большей частотой подавляются сильнее, поэтому уже при переходе ко второй секции форма сигнала на ней приближается к форме входного сигнала трансформатора. Высокочастотные колебания, наблюдаемые в сигналах со второй и третьей секций, обусловлены наличием конструктивной емкости между витками обмоток и возбуждаются в момент закрытия моста. При открытии моста и увеличении нагрузки эти высокочастотные колебания затухают. Паразитные колебания достаточно высокочастотны (1-2 MHz) и могут быть легко отфильтрованы входными цепями вторичных источников питания. Таким образом, минимизируется возможность получить проблемы, связанные с высокочастотными паразитными резонансами на собственной межвитковой емкости. Такого рода проблемы наблюдались в конструкции каскадного трансформатора, описанного в работе [15] и не позволившие ему выйти на рабочие параметры.

Также с использованием измеренного входного сигнала трансформатора проводилось моделирование переходных процессов в трансформаторе и сравнение результатов моделирования с измерениями. На рис. $13, b$ жирными точками обозначены моделированные значения напряжения на третьей измерительной обмотке, базирующиеся на измеренном сигнале с первой секции при нагрузке трансформатора $R_{\mathrm{LOAD}}=8 \Omega$. Расчетные значения хорошо ложатся на измеренные кривые, поэтому можно заключить, что используемая модель трансформатора является правдоподобной, и параметры трансформатора определены корректно. Далее эти параметры использовались для моделирования трансформатора, состоящего из десяти секций, и расчетов его эффективности, как устройства для передачи мощности.

\section{3. Эффективность каскадного трансформатора с объемным витком}

Наличие индуктивности рассеяния приводит к просадке напряжения вдоль секций трансформатора. Вывод 


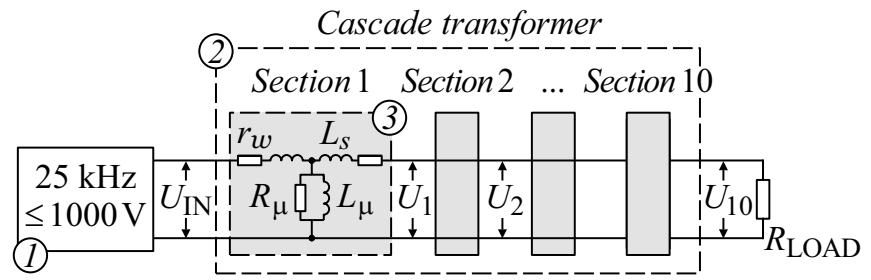

Рис. 14. Моделируемая схема каскадного трансформатора из десяти секций: 1 - источник синусоидального сигнала с амплитудой напряжения до $1000 \mathrm{~V}, 2$ - каскадный трансформатор, 3 - секция каскадного трансформатора. $U_{\mathrm{IN}}-$ входное напряжение трансформатора, $U_{1}, \ldots, U_{9}-$ напряжение на выходе соответствующих секций, $U_{10}-$ напряжение на нагрузке $R_{\text {LOAD. }}$

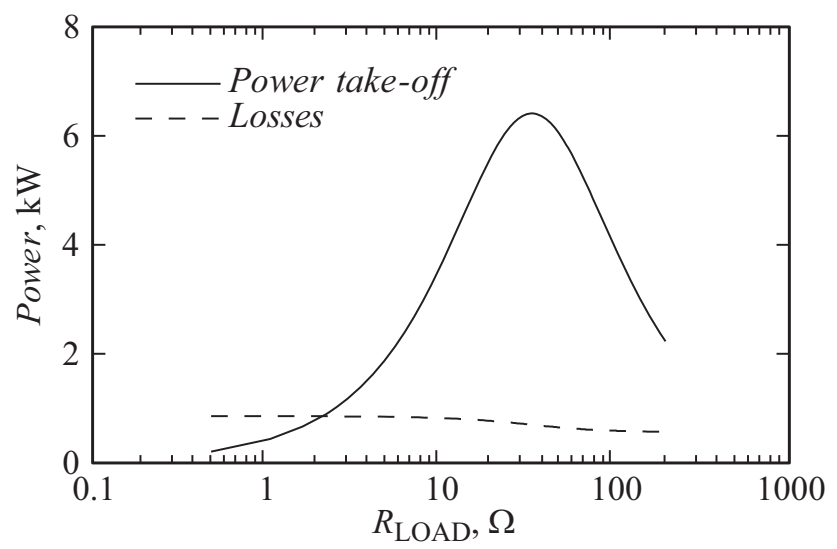

Рис. 15. Величина мощности, отбираемой нагрузкой и теряемой в секциях трансформатора, в зависимости от величины нагрузки.

о пригодности трансформатора для конкретной задачи можно сделать, рассчитав максимальную мощность, которую данный трансформатор может передать. Для дальнейшего описания трансформатора используется линейная модель, в которой потребителем мощности выступает сопротивление $R_{\mathrm{LOAD}}$ (рис. 14). Напряжение источника питания считается фиксированным. Поскольку параметры секций трансформатора мало отличаются при переходе от секции к секции, в моделируемой схеме полагается, что все секции одинаковы и могут быть представлены каждая симметричной Т-образной схемой.

На рис. 15 представлена зависимость отбираемой мощности от сопротивления нагрузки при рабочей частоте $25 \mathrm{kHz}$, а также ее сравнение с суммарными потерями в трансформаторе. Для амплитудного напряжения источника $1000 \mathrm{~V}$ (среднеквадратичное $700 \mathrm{~V}$ ), максимальное значение отбираемой мощности составляет $6.4 \mathrm{~kW}$. Наличие максимума следует из того, что трансформатор и внешний источник питания образуют генератор с некоторым выходным импедансом, а максимальная отбираемая от данного генератора мощность достигается при согласовании выходной нагрузки с выходным импедансом.
Ограничение по выходной мощности в данном случае обусловлено просадкой напряжения от секции к секции из-за индуктивности рассеяния секций. Максимальное значение передаваемой мощности зависит от напряжения источника питания и меняется вместе с ним пропорционально квадрату напряжения (для линейной модели). Однако максимальное рабочее напряжение, в свою очередь, ограничено максимально допустимым напряжением на используемых силовых элементах, включенных в схемы отбора мощности с секций трансформатора.

Для повышения эффективности трансформатора необходимо компенсировать просадку напряжения, обусловленную наличием индуктивности рассеяния секций. Так как в рассматриваемой модели связь между соседними секциями трансформатора осуществляется при помощи единичных витков, охватывающих обе секции, добавить емкость для компенсации индуктивности рассеяния в каждую секцию не представляется возможным. Решением является установка набора компенсирующих конденсаторов в начало или в конец трансформатора, или с обеих сторон одновременно.
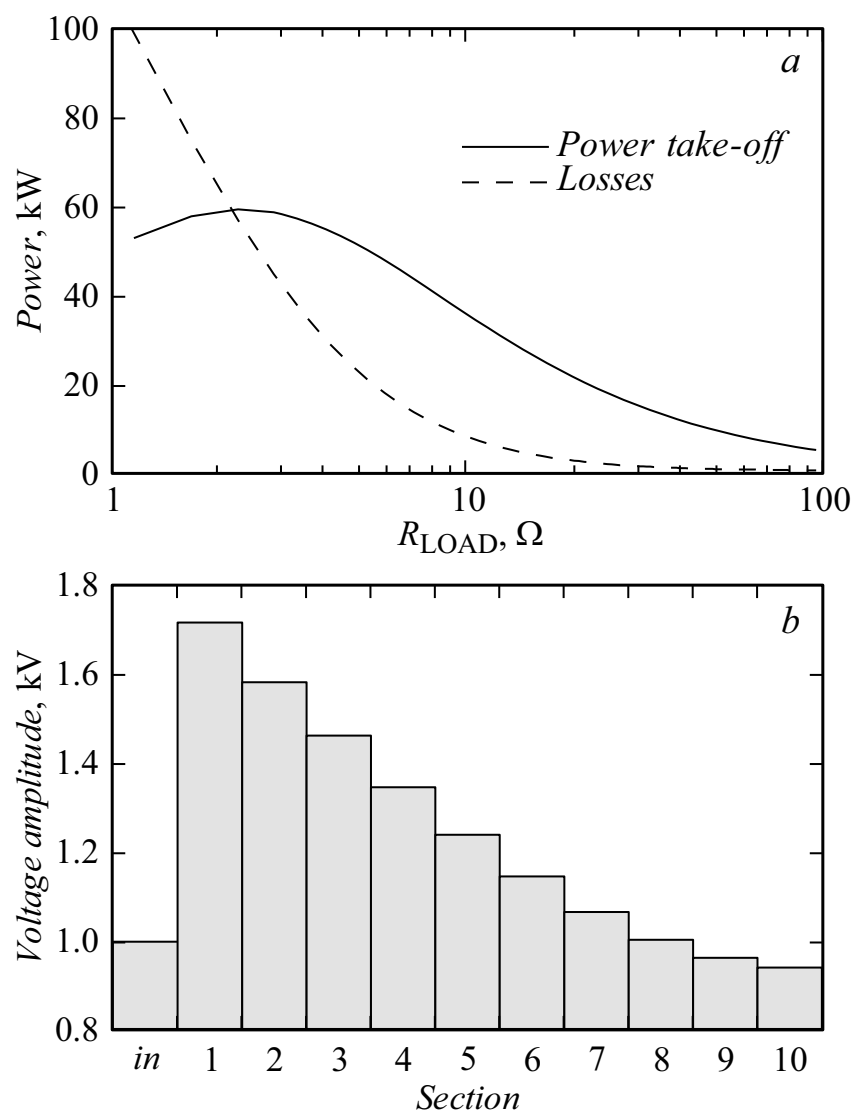

Рис. 16. Передаточные характеристики каскадного трансформатора из 10 секций при подключении компенсирующего конденсатора $C_{S}=180 \mathrm{nF}$ ко входу трансформатора: $a-$ величина мощности отбираемой нагрузкой и теряемой в секциях трансформатора в зависимости от величины нагрузки, $b-$ амплитуда напряжения на выходе секций трансформатора, „in“ - напряжение источника питания. 

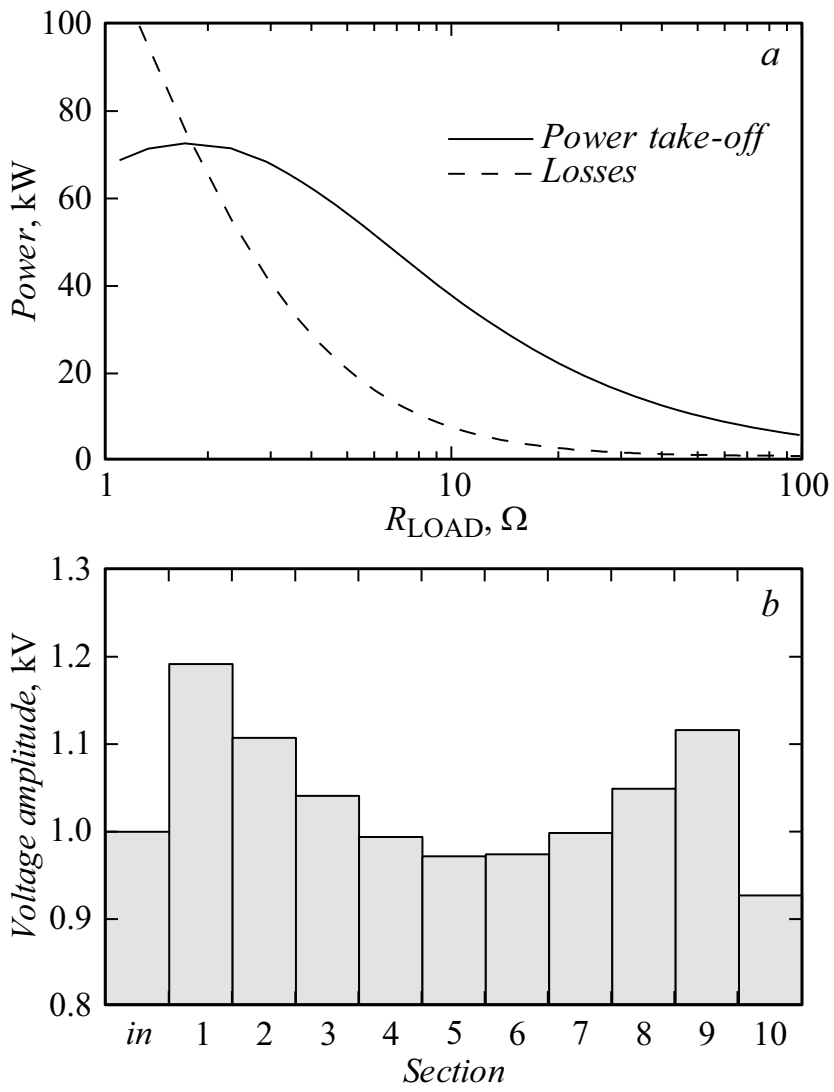

Рис. 17. Передаточные характеристики каскадного трансформатора из 10 секций при симметричном подключении компенсирующих емкостей ко входу и выходу трансформатора: $a-$ величина мощности, отбираемой нагрузкой и теряемой в секциях трансформатора, в зависимости от величины нагрузки, $b$ - амплитуда напряжения на выходе секций трансформатора, „in“ - напряжение источника питания.

При размещении компенсирующего конденсатора $C_{S}=180 \mathrm{nF}$ на входе трансформаторного каскада кривая отбора мощности приобретает вид, изображенный на рис. $16, a$, а также распределение напряжения вдоль секций трансформатора при отборе мощности $20 \mathrm{~kW}$ (рис. $16, b)$. В данном случае выходное сопротивление трансформатора определяется сопротивлением проводов обмоток, значение которого много меньше значения импеданса, обусловленного индуктивностью рассеяния трансформатора.

В рассматриваемой цепи конденсатор используется для компенсации падения напряжения между трансформаторными секциями, обусловленного наличием индуктивности рассеяния. Как следствие, на данном элементе наблюдаются большие падения напряжения. При передаче мощности $20 \mathrm{~kW}$ амплитуда входного тока трансформатора составляет $45 \mathrm{~A}$. При рабочей частоте $25 \mathrm{kHz}$ падение напряжения на компенсирующем конденсаторе составляет $1.6 \mathrm{kV}$.

Неравномерность распределения напряжения вдоль секций трансформатора может быть уменьшена, если разместить компенсирующие конденсаторы не с одной стороны трансформатора, а с обеих сторон. Номинал емкости с каждой стороны выбирается равным $360 \mathrm{nF}$, при этом падение напряжения на конденсаторах с каждой стороны составляет $0.8 \mathrm{kV}$. Также при этом возрастает максимальное возможное значение отбираемой мощности $70 \mathrm{~kW}$ при амплитуде входного сигнала источника $1000 \mathrm{~V}$ (рис. $17, a$ ). На рис. $17, b$ амплитуда напряжения на 10-й секции указана с учетом того, что компенсирующая емкость включена в эту секцию.

\section{Заключение}

Ключевым аспектом настоящей работы является обзор системы передачи мощности устройствам, находящимся под высоким потенциалом в высоковольтной колонне охладителя для SRing. В качестве устройства для передачи и распределения мощности предлагается использовать каскадный трансформатор, схожий с трансформатором, используемым в установке электронного охлаждения для COSY [11]. Отличительной особенностью трансформатора, рассматриваемого в настоящей работе, является отсутствие конденсаторов между его секциями, используемых для компенсации индуктивности рассеяния обмоток. Подобная модификация позволяет значительно упростить проектирование трансформатора, а также процесс его сборки и эксплуатации. Однако измерения прототипа каскадного трансформатора и расчет эффективности передачи мощности показывают, что для передачи больших мощностей полностью отказаться от компенсации индуктивности рассеяния трансформатора нельзя. В случае относительно небольшого числа секций возможно ограничиться добавлением компенсирующих конденсаторов только на входе и выходе каскадного трансформатора. Симметричное распределение компенсирующих емкостей позволяет максимально снизить перепады напряжений между соседними секциями, обеспечивая более регулярное распределение напряжений.

Работа выполнена при поддержке фонда РФФИ (грант № 16-52-53016), National Natural Science Foundation of China (N 11575264, 11375245, 11475235, 11611130016) и Hundred Talents Project of the Chinese Academy of Sciences.

\section{Список литературы}

[1] Mao L.J., Yang J.C., Xia J.W., Yang X.D., Yuan Y.J., Li J., Ma X.M., Yan T.L., Yin D.Y., Chai W.P., Sheng L.N., Shen G.D., Zhao H., Tang M.T. // Nucl. Instrum. Meth. Phys. Res. 2015. Section A: Vol. 786. P. 91-96.

[2] Пархомчук В.В., Скринский А.Н. // УФН. 2000. Т. 170. C. 473-493. 
[3] Antokhin E.I., Bocharov V.N., Bublei A.V., Goncharov A.D., Grishanov B.I., Konstantinov E.S., Konstantinov S.G., Krainov G.S., Kudryavtsev A.M., Kuksanov N.K., Kuznetsov G.M., Logatchov P.V., Myakishev D.G., Nemytov P.I., Panasyuk V.M., Parkhomchuk V.V., Reva V.B., Salimov R.A., Scarbo B.A., Smirnov B.M., Sukhina B.N., Tupikov V.S., Veis M.E. // Nucl. Instrum. Meth. Phys. Res. 2009. Section A: Vol. 441. P. 87-91.

[4] Parkhomchuk V., Bocharov V., Brizgunov M., Bubley A., Evtuchenko Yu., Goncharov A., Ivanov A., Kokoulin V., Kolmogorov V., Kondaurov M., Konstantinov S., Kozak V., Krainov G., Kruchkov A., Kuper E., Medvedko A., Mironenko L., Reva V., Shrainer K., Skarbo B., Skrinsky A.N., Smirnov B., Sukhina B., Vedenev M., Voskoboinikov R., Zakhvatkin M., Zapiatkin N., Xiaodong Yang, Hong Wey Zhao // AIP Conf. Proceed. 2006. Vol. 821. P. 334-340. DOI: 10.1063/1.2190130

[5] Bocharov V., Bubley A., Boimelstein Yu., Veremeenko V., Voskoboinikov V., Goncharov A., Grishanov V., Dranichnikov A., Evtushenko Yu., Zapiatkin N., Zakhvatkin M., Ivanov A., Kokoulin V., Kolmogorov V., Kondaurov M., Konstantinov E., Konstantinov S., Krainov G., Kriuchkov A., Kuper E., Medvedko A., Mironenko L., Panasiuk V., Parkhomchuk V., Petrov S., Reva V., Svischev P., Skarbo B., Smirnov B., Sukhina B., Tiunov M., Shirokov V., Shrainer K., Yang X.D., Zhao H.W., Wang Z.X., Li J., Zhang J.H., Zhang W., Yan H.B., Yan H.H., Xia G.X. // Nucl. Instrum. Methods Phys. Res. 2004. Section A: Vol. 532. P. 144-149.

[6] Бочаров В.Н., Бублей А.В., Боймельштейн Ю.М., Веремеенко В.Ф., Воскобойников Р.В., Гончаров А.Д., Гришанов Б.И., Драничников А.Н., Евтушенко Ю.А., Заплткин Н.П., Захваткин М.Н., Иванов А.В., Кокоулин В.И., Колмогоров В.В., Кондауров М.Н., Константинов Е.С., Константинов С.Г., Крайнов Г.С., Крючков А.М., Купер Э.А., Медведко А.С., Мироненко Л.А., Панасюк В.М., Пархомчук В.В., Петров С.П., Рева В.Б., Свищев П.В., Скарбо Б.А., Смирнов Б.М., Сухина Б.Н., Тиунов М.А., Широков В.В., Шрайнер К.К., Янг С., Хонгвей Джао // Материалы конференции RUPAC-2002. 2004. Т. 2. С. 699 704.

[7] Валяев Ю.Д., Казарезов И.В., Кузнецов В.И., Останин В.П. Малогабаритный высокочастотный разделительный трансформатор для питания устройств расположенных под высоким потенциалом. Препринт 89-160. ИЯФ СО РАН, Новосибирск, 1989. 25 с.

[8] Veremeenko V.M., Voskoboynikov R.V., Goncharov A.D. // Proc. RuPAC XX. 2006. P. 97-99.

[9] Behtenev E., Bocharov V., Bubley V. // Proc. RuPAC XIX. 2004. P. 506-510.

[10] Салимов Р.А. // УФН. 2000. Т. 170. № 2. С. 197-201.

[11] Брызгунов М.И., Гончаров А.Д., Панасюк В.М., Пархомчук В.В., Рева В.Б., Скоробогатов Д.Н. // Приборы и техника эксперимента. 2015. № 2. С. 14-23.

[12] Kazakevich G., Burov A., Boffo C. Recycler Electron Cooling project: Mechanical vibrations in the Pelletron and their effect on the beam. Preprint FERMILAB-TM-2319-AD, 2005.

DOI: $10.2172 / 15020252$

[13] Hofmann A., Aulenbacher K., Bruker M.-W., Dietrich J., Friederich S., Weilbach T. Proceedings of COOL-2013, 2013. P. 107-109.
[14] Алиновский Н.И., Гончаров А.Д., Клюев В.Ф., Константинов С.Г., Константинов Е.С., Крючков А.М., Пархомчук В.В., Петриченков М.В., Растигеев С.А., Рева В.Б. // ЖТФ. 2009. Т. 79. Вып. 9. С. 10-7111.

[15] Reginato L. Proc. PAC-1991. P. 2918-2922. 